\title{
A CLASSIFICATION OF THE STRUCTURALLY STABLE CONTRACTING ENDOMORPHISMS OF $S^{1}$
}

\author{
LOUIS BLOCK AND JOHN FRANKE
}

\begin{abstract}
An open dense set of contracting endomorphisms of $S^{1}$, the circle, are found to be structurally stable. This set is classified up to topological conjugacy by a countable number of invariants.
\end{abstract}

1. In this paper we prove necessary and sufficient conditions for contracting $C^{2}$ endomorphisms of $S^{1}$, i.e. $C^{2}$ maps with $\left|d f_{x}\right|<1$, to be structurally stable in the $C^{2}$-topology. Shub in [4] proved structural stability for expanding endomorphisms. In [5], Smale asks for a characterization of the structurally stable endomorphisms of $S^{1}$. Nitecki has proven a structural stability theorem for nonsingular endomorphisms of $S^{1}$ in [2]. It should be noted that this paper contains a basic error in the proof of the density of Axiom A. We have just received a preprint from Nitecki [3] in which he constructs Markov partitions for a general class of endomorphisms of degree greater than one, and proves a structural stability theorem. M. V. Jakobson in [6], which has been translated since the writing of this paper, constructs an open dense set of endomorphisms in $C^{1}\left(S^{1}, S^{1}\right)$ which are $\Omega$-stable, and shows that a certain subset of these are structurally stable in $C^{2}\left(S^{1}, S^{1}\right)$.

We will let $K$ be the set of contracting $C^{2}$ endomorphisms of $S^{1}$ with the $C^{2}$ topology. If $f \in K$, then $x_{0}(f)$ will denote its unique fixed point. Let $K_{1}$ be the subset of $K$ consisting of those functions $f$ with the property that $d f_{x}=0$ implies $d^{2} f_{x} \neq 0$. If $f \in K_{1}$, then $f$ has only a finite number of points with $d f_{x}=0$. This is because any limit would have $d f_{x}=0$ and $d^{2} f_{x}=0$. The points in the set $T(f)=\left\{x \in S^{1}\right.$ with $\left.d f_{x}=0\right\}$ will be called turning points.

The orbit of $x$ will be denoted $\operatorname{orb}(x)$ and defined to be $\left\{f^{n}(x): n \geqq 0\right\}$. Let $K_{2}$ be those functions in $K$ such that $x_{0}(f) \notin \operatorname{orb}(x)$ for any $x \in \bar{T}(f)$. The set of $f$ in $K$ with the property that $x, y \in T(f)$ and $\operatorname{orb}(x) \cap \operatorname{orb}(y) \neq \varnothing$ imply $x=y$ will be denoted by $K_{3}$. Let $K_{0}=K_{1} \cap K_{2} \cap K_{3}$. In $\S 2$, we prove that $K_{0}$ is open and dense in $K$. The functions in $K_{0}$ are classified up to

Received by the editors February 2, 1972.

AMS (MOS) subject classifications (1970). Primary 58F10, 58F15.

Key words and phrases. Contracting endomorphism, structural stability, topological conjugacy.

(c) American Mathematical Society 1973 
topological conjugacy in $\S 3$. Finally in $\S 4$, we prove that $K_{0}$ is precisely those elements in $K$ which are structurally stable in the $C^{2}$ topology.

We will use the notation $[a, x, b]$ to denote the arc from $a$ to $b$ containing $x$, and $\left[a,(x)^{*}, b\right]$ to denote the arc from $a$ to $b$ not containing $x$.

We would like to thank J. Franks and R. F. Williams for the ir encouragement.

\section{Lemma 1. $K_{1}$ is open and dense in $K$.}

Proof. Density follows from a standard transversality theorem (see [1]). Openness is also well known but we give a simple proof so that we can refer to it later. So let $f \in K_{1}$.

About each point $x \in T(f)$, pick an open interval $\left(a_{x}, b_{x}\right)$ such that $d^{2} f_{y} \neq 0$ for all $y$ in the closed interval $\left[a_{x}, b_{x}\right]$. Let $T=S^{1}-\cup\left(a_{x}, b_{x}\right)$, where the union is taken over $x \in T(f)$. Then if $\alpha=\min \left\{\left|d f_{y}\right|: y \in T\right\}$, $\alpha \neq 0$.

Hence by perturbations less than $\alpha$ we cannot create points where $d f_{y}=0$, outside of $\bigcup\left(a_{x}, b_{x}\right)$.

Let $\beta=\min \left\{\left|d f_{z}\right|: z=a_{x}\right.$ or $z=b_{x}$ for some $\left.x \in T(f)\right\}$. Since $d^{2} f$ is either positive or negative on each interval $\left[a_{x}, b_{x}\right]$, df is either increasing or decreasing. Thus $\beta>0$.

Let $\gamma=\inf \left\{\left|d^{2} f_{z}\right|: z \in \bigcup\left[a_{x}, b_{x}\right]\right\}$. Then $\gamma>0$.

Now a perturbation of less than $\min \{\gamma, \beta\}$ must have exactly one point $y \in\left(a_{x}, b_{x}\right)$ with $d f_{y}=0$. Thus a perturbation of less than $\min \{\alpha, \gamma, \beta\}$ will still be in $K_{1}$. Q.E.D.

LeMma 2. $K_{2}$ is open and dense in $K_{1}$.

Proof (Openness). Assume $f \in K_{1}$ and satisfies $d f_{x}=0$ implies $x_{0}(f) \notin$ $\operatorname{orb}(x)$. We must show perturbations of $f$ have this property. Let $J$ be a closed interval containing $x_{0}(f)$ such that if $x \in J, d f_{x} \neq 0$ and such that $f$ maps $J$ injectively into its interior, $I$. Then $\exists N$ such that $f^{N}(x) \in I, \forall x \in T(f)$ (since $T(f)$ is finite and $f$ is a contraction). If $g$ is close enough to $f, x_{0}(g)$ is close to $x_{0}(f), g^{N}(x) \in I$ with $g^{N}(x) \neq x_{0}(g)$, $\forall x \in T(g)$, and no $x \in T(g)$ is in $I$. Here we are using the fact that card $T(f)=$ card $T(g)$ and the elements of $T(f)$ are near the elements of $T(g)$ which follows from the proof of Lemma 1 . But since $I$ is mapped 1-1 into itself by $g$, with $x_{0}(g)$ fixed, $g^{m}(x) \neq x_{0}(g)$ for any $m$ if $x \in T(g)$.

(Density). Let $f \in K_{1}$. If $d f\left(x_{0}(f)\right)=0$, then by an arbitrarily small rotation we can perturb $f$ so $d f\left(x_{0}(f)\right) \neq 0$. Order the turning points of $f, x_{1}, \cdots, x_{n}$, such that $d\left(x_{i}, x_{0}(f)\right) \leqq d\left(x_{i+1}, x_{0}(f)\right)$ where $d$ is the usual metric on $S^{1}$. Suppose $x_{0}(f) \in \operatorname{orb}\left(x_{1}\right)$. Let $I_{1}$ be any small interval about $x_{1}$, and $m=\min \left\{n \geqq 1: f^{n}\left(x_{1}\right)=x_{0}(f)\right\}$. We can perturb $f$ in $I_{1}$ so that $f^{m}\left(x_{1}\right) \neq x_{0}(f)$, but $f^{m}\left(x_{1}\right)$ is in a neighborhood of $x_{0}(f)$ in which $f$ is $1-1$. 
Hence $x_{0}(f) \notin \operatorname{orb}\left(x_{1}\right)$. Then let $I_{2}$ be a small interval about $x_{2}$ which does not contain any iterates of $x_{1}$. (This is possible since $f$ is a contraction.) We can perturb $f$ on $I_{2}$ so that $x_{0}(f) \notin \operatorname{orb}\left(x_{2}\right)$ without affecting iterates of $x_{1}$, so that we still have $x_{0}(f) \notin \operatorname{orb}\left(x_{1}\right)$. Repeating the process $n$ times, we perturb $f$ to a map in $K_{2}$. Q.E.D.

Lemma 3. $K_{3}$ is open and dense in $K_{1} \cap K_{2}$.

Proof. First we make a few definitions which will be used in this proof and also later in the paper. Let $f \in K_{1} \cap K_{2}$. Let $I$ be a symmetric closed interval $\left[a, x_{0}(f), b\right]$, about $x_{0}(f)$, in which there are no turning points. For each nonnegative integer $j$ let

$$
I_{j}(f)=\left(f^{j}\left[a, x_{0}(f), b\right]\right)-\left(f^{j+1}\left[a, x_{0}(f), b\right]\right) .
$$

Note that $I_{j}(f)$ is essentially just a fundamental domain for $f$.

Then $\exists j$ such that, $\forall x_{i} \in T(f), \exists n(i) \geqq 0$ with $f^{n(i)}\left(x_{i}\right) \in I_{j}(f)$, since $f$ is a contraction and $f \in K_{2}$. Let $j(f)$ be the smallest such $j$. Let $M(f)$ be the smallest number $M$ such that, $\forall x_{i} \in T(f), \exists n(i) \leqq M$ with $f^{n(i)}\left(x_{i}\right) \in$ $I_{j(f)}(f)$.

(Openness). Let $f \in K_{3} \cap K_{1} \cap K_{2}$. Let $g$ be a perturbation of $f$ in $K_{1} \cap K_{2}$. We must show $g \in K_{3}$. By the proof of Lemma 1, we can make the perturbation small enough so that the elements of $T(g)$ lie arbitrarily close to the elements of $T(f)$. Hence we can make the first $M(f)$ iterates of $g$ close to the first $M(f)$ iterates of $f$, preventing any intersections in the first $M(f)$ iterates of $T(g)$ under $g$, and ensuring that $j(f)=j(g)$ and $M(f)=M(g)$. But $I_{j(g)}$ is mapped $1-1$ onto $I_{j(g)+1}$, so by the definition of $j(g)$ there are no intersections of orbits of points in $T(g)$.

(Density). Let $f \in K_{1} \cap K_{2}$. Let $T(f)=\left\{x_{1}, \cdots, x_{n}\right\}$ be ordered as in Lemma 2. Let $j(f)$ and $M(f)$ be as above. First perturb $f$ in a neighborhood of $x_{n}$ so that $\left\{f^{m}\left(x_{n}\right): m \leqq M(f)\right\}$ does not contain any of the first $M(f)$ iterates of $\left\{x_{1}, \cdots, x_{n-1}\right\}$. After the first perturbation $\exists$ a neighborhood of $x_{n-1}$ which does not contain any iterates of other turning points. So we can perturb $f$ in this neighborhood so that $\left\{f^{m}\left(x_{n-1}\right): m \leqq M(f)\right\}$ does not contain any of the first $M(f)$ iterates of $\left\{x_{1}, \cdots, x_{n-2}, x_{n}\right\}$. Repeating the process we perturb $f$ to a function with no intersections in the first $M(f)$ iterates of its turning points. By choice of $M(f)$, this completes the proof. Q.E.D.

Combining Lemmas 1,2 , and 3 we have

THEOREM 1. $K_{0}$ is open and dense in $K$.

3. Lemma 4. If $h$ is a conjugacy between $f$ and $g \in K_{0}$ and $x \in T(f)$, then $h(x) \in T(g)$. 
Proof. Let $x \in T(f)$ and suppose $h(x) \notin T(g)$. Then $\exists$ an interval $I$ containing $h(x)$ on which $g$ is $1-1$, by the inverse function theorem. Pick $a, b \in h^{-1}(I)$ with $f(a)=f(b)$. Then $h(f(a))=h(f(b))$ but $g(h(a)) \neq g(h(b))$, a contradiction. Q.E.D.

By exactly the same proof, we have

COROLlaRY 1. If $h$ is a conjugacy between $f$ and $g \in K$, and $x \in T(f)$ has the property that no open interval containing $x$ is mapped $1-1$ by $f$, then $h(x)$ is a turning point of $g$ with the same property.

Definition. Let $f \in K_{0}$. Label the elements of $T(f), x_{1}, \cdots, x_{n}$, starting counterclockwise from the fixed point. We will use this canonical ordering for the remainder of this paper. (When we are dealing with $f$ and $g \in K_{0}$, we will let $T(f)=\left\{x_{1}, \cdots, x_{n}\right\}$ and $T(g)=\left\{y_{1}, \cdots, y_{n}\right\}$ with this ordering. We will also let $x_{0}(f)=x_{0}, x_{6}(g)=y_{0}$.) Let $\operatorname{orb}(T(f))=$ $\left\{f^{k}\left(x_{l}\right): x_{l} \in T(f), k \geqq 0\right\}$. We order orb $(T(f))$ by defining:

$Z_{1}^{\prime}(f)=x_{1}$,

$Z_{2}^{\prime}(f)=$ the closest element of $\operatorname{orb}(T(f))$ to $x_{1}$ in $\left(x_{1},\left(x_{n}\right)^{*}, x_{0}\right]$, and assuming $Z_{n-1}^{\prime}(f)$ is defined, we let

$Z_{n}^{\prime}(f)$ be the closest element of $\operatorname{orb}(T(f))$ to $Z_{n-1}^{\prime}(f)$ in $\left(Z_{n-1}^{\prime}(f)\right.$, $\left.\left(x_{n}\right)^{*}, x_{0}\right]$.

We also let:

$Z_{-1}^{\prime}(f)=$ the closest element of $\operatorname{orb}(T(f))$ to $x_{1}$ in $\left(x_{1},\left(Z_{2}^{\prime}(f)\right)^{*}, x_{0}\right]$, and assuming $Z_{-n+1}^{\prime}(f)$ is defined, let

$Z_{-n}^{\prime}(f)=$ the closest element of orb $(T(f))$ to $Z_{-n+1}^{\prime}(f)$ in $\left(Z_{-n+1}^{\prime}(f)\right.$, $\left.\left(Z_{2}^{\prime}(f)\right)^{*}, x_{0}\right]$.

In other words, we number orb $(T(f))$ positively in one direction from $x_{1}$, and negatively in the other. This makes sense, since $x_{0}$ is the only accumulation point of $f$, and $\exists$ an infinite sequence of elements of $\operatorname{orb}(T(f))$ on each side of $x_{0}$ approaching $x_{0}$.

Let $Z_{i}(f)=(r(i), k(i))$ where $Z_{i}^{\prime}(f)=f^{r(i)}\left(x_{k(i)}\right)$.

Define $Z_{i}^{\prime}(f)$ and $Z_{i}(f)$ by the same formal definition as above, except number the turning points clockwise from the fixed point.

THEOREM 2. Let $f, g \in K_{0} . f$ and $g$ are topologically conjugate iff $Z_{i}(f)=Z_{i}(g), \forall i$, or $Z_{i}(f)=Z_{i}(g), \forall i$.

Proof. First suppose $Z_{i}(f)=Z_{i}(g), \forall i$. Then $f$ and $g$ are both orientation preserving or reversing at their fixed points. If $f$ is orientation preserving, let $I(f)=\left[x_{1}, x_{0}, x_{n}\right], I(g)=\left[y_{1}, y_{0}, y_{n}\right]$. If $f$ is orientation reversing and $f\left(x_{n}\right)=Z_{i}(f)$ with $i>0$, let $I(f)=\left[x_{n}, x_{0}, f\left(x_{n}\right)\right]$ and $I(g)=$ $\left[y_{n}, y_{0}, g\left(y_{n}\right)\right]$. If $i<0$, let $I(f)=\left[x_{1}, x_{0}, f\left(x_{1}\right)\right]$ and $I(g)=\left[y_{1}, y_{0}, g\left(y_{1}\right)\right]$. Let $a$ be the endpoint of $I(f)$ in the counterclockwise direction from $x_{0}$ and let $b$ be the other endpoint. Then $j(f)=j(g)$, where $j(f)$ is as defined 
in Lemma 3. Here of course we use the intervals we have just defined for the $I$ in Lemma 3.

Take any homeomorphism $h: I_{j(f)}(f) \rightarrow I_{j(g)}(g)$ sending any $Z_{i}^{\prime}(f)$ in $I_{j(f)}(f)$ to $Z_{i}^{\prime}(g)$, which is possible since $Z_{i}(f)=Z_{i}(g)$. Define $h$ on each $I_{k}(f)$ by $h(x)=g^{k-j(g)} h f^{j(f)-k}(x)$. Here we only look at $I(f)$ and $I(g)$, so negative iteration makes sense. Setting $h\left(x_{0}\right)=y_{0}$ we get a conjugacy from $I(f)$ to $I(g)$. Let $a_{1}$ be the first point not in $I(f)$ in the counterclockwise direction from $I(f)$ that gets mapped to $a$ or $b$. Let $b_{1}$ be the first point not in $I(f)$ in the clockwise direction from $I(f)$ that gets mapped to $a$ or $b$.

Case 1. If $\exists$ no such point $a_{1}$, then $f\left(S^{1}\right) \subset I(f)$. We extend $h$ to $S^{1}$ by mapping $\left[x_{k},\left(x_{0}\right)^{*}, x_{k+1}\right] \rightarrow\left[y_{k},\left(y_{0}\right)^{*}, y_{k+1}\right]$ by $h(x)=g^{-1} h f(x)$, where the inverse is taken in $\left[y_{k},\left(y_{0}\right)^{*}, y_{k+1}\right]$ as $g$ is $1-1$ on $\left[y_{k},\left(y_{0}\right)^{*}, y_{k+1}\right]$. Note that $h\left(x_{k}\right)=y_{k}$ since $h\left(Z_{i}^{\prime}(f)\right)=Z_{i}^{\prime}(g), \forall i$. In the orientation reversing case, one of the intervals $\left[a,\left(x_{0}\right)^{*}, x_{1}\right)$ or $\left[b,\left(x_{0}\right)^{*}, x_{n}\right)$ is nonempty and we define $h$ on this interval by the same formula.

Case 2. If $\exists a_{1}$ as above, then also $\exists b_{1}$ and we extend $h$ to $\left[b_{1}, x_{0}, a_{1}\right]$ by defining $h$ separately on the intervals $\left[x_{1},\left(x_{0}\right)^{*}, x_{2}\right],\left[x_{2},\left(x_{0}\right)^{*}, x_{3}\right], \cdots$, $\left[x_{p},\left(x_{0}\right)^{*}, a_{1}\right]$, where $x_{2}, \cdots, x_{p} \in\left[x_{1},\left(x_{0}\right)^{*}, a_{1}\right]$, and on the intervals $\left[x_{n},\left(x_{0}\right)^{*}, x_{n-1}\right],\left[x_{n-1},\left(x_{0}\right)^{*}, x_{n-2}\right], \cdots,\left[x_{n-q},\left(x_{0}\right)^{*}, b_{1}\right]$ where $x_{n-1}, \cdots$, $x_{n-a} \in\left[x_{n},\left(x_{0}\right)^{*}, b_{1}\right]$ as in Case 1 . In the orientation reversing case, if there is an interval of the form $\left[a,\left(x_{0}\right)^{*}, x_{1}\right)$ or $\left[b,\left(x_{0}\right)^{*}, x_{n}\right)$ in $\left[b_{1}, x_{0}, a_{1}\right]$, we define $h$ there, also. In any case $h$ sends $Z_{i}^{\prime}(f)$ to $Z_{i}^{\prime}(g)$.

Next we define $a_{2}, b_{2}$ analogously to $a_{1}, b_{1}$ using $\left[b_{1}, x_{0}, a_{1}\right]$ instead of $I(f)$ and extend $h$ to $\left[b_{2}, x_{0}, a_{2}\right]$. Since $f$ is a contraction, eventually there will exist no $a_{n}$ and we can extend $h$ to all of $S^{1}$ as in Case $1 . h$ is clearly a conjugacy.

If $Z_{i}(f)=Z_{i}(g)$ then again $f$ and $g$ are both orientation preserving or reversing at their fixed points. We can define $I(f)$ and $I(g)$ as above, with $j(f)=j(g)$. We define a homeomorphism $h$ on $I_{j(f)}$ mapping $Z_{i}^{\prime}(f)$ to $Z_{i}^{\prime}(g)$ and then extend $h$ as above. This proves necessity.

Now, conversely, let $h$ be a conjugacy between $f$ and $g$. By Lemma 4, $h(T(f))=T(g)$. Also $h\left(x_{0}\right)=y_{0}$. Hence, either $h\left(x_{n}\right)=y_{n}$ or $h\left(x_{n}\right)=y_{1}$.

Case 1. $h\left(x_{n}\right)=y_{n}$. Then $h\left(x_{i}\right)=y_{i}, \forall i$. Note that $h\left(Z_{i}^{\prime}(f)\right)=Z_{i}^{\prime}(g), \forall i$, since iterates of turning points are preserved by $h$ and $h^{-1}$. But

$$
h\left(Z_{i}^{\prime}(f)\right)=h\left(f^{r(i)}\left(x_{k(i)}\right)\right)=g^{r(i)}\left(h\left(x_{k(i)}\right)\right)=g^{r(i)}\left(y_{k(i)}\right) .
$$

Hence $g^{r(i)}\left(y_{k(i)}\right)=Z_{i}^{\prime}(g)$, so $Z_{i}(g)=(r(i), k(i))=Z_{i}(f)$.

Case 2. $h\left(x_{n}\right)=y_{1}$. Then renumber the turning points (counting clockwise) so that $h\left(x_{n}\right)=y_{n}$. Then $h\left(x_{i}\right)=y_{i}, \forall i$, and it follows exactly as in Case 1 that $Z_{i}(f)=Z_{i}(g), \forall i$. Q.E.D.

4. THEOREM 3. $f \in K_{0}$ implies $f$ is structurally stable. 
Proof. Let $g$ be a perturbation of $f$. Since $K_{0}$ is open we can assume $g \in K_{0}$. We can also assume card $T(f)=$ card $T(g)$ by the proof of Lemma 1. Pick $M(f)$ and $j(f)$ as in Lemma 3. We can make the perturbation small enough so that the first $M(f)$ iterates of $T(g)$ lie arbitrarily close to the first $M(f)$ iterates of $T(f)$. Thus we can ensure that whenever $Z_{i}^{\prime}(f) \in$ $I_{k}(f)$ we have $Z_{i}^{\prime}(g) \in I_{k}(g)$ and $Z_{i}(f)=Z_{i}(g), \forall k \leqq j(f)$. But this implies $j(f)=j(g)$ and $Z_{i}(f)=Z_{i}(g), \forall i$. Hence by Theorem $2, f$ and $g$ are topologically conjugate. Q.E.D.

THEOREM 4. If $f \in K$ is structurally stable, then $f \in K_{0}$.

PROOF. If $d f_{x}=0$ and $d^{2} f_{x}=0$ then by an arbitrarily small perturbation we could increase the number of turning points which have the property that $f$ is not $1-1$ on any interval containing that point. But $f$ must have a finite number of such turning points by Corollary 4 , since $f$ is conjugate to some $f^{\prime} \in K_{0}$ by hypothesis and Theorem 1. This contradicts the structural stability of $f$. Hence $f \in K_{1}$.

Suppose $d f_{x}=0, x_{0}(f) \in \operatorname{orb}(x)$. By the first paragraph, $d^{2} f_{x} \neq 0 . f$ must be conjugate to some $f^{\prime} \in K_{0}$. But a conjugacy must send $x_{0}(f)$ to a point which is both fixed and on the orbit of a turning point. This contradicts $f^{\prime} \in K_{0}$; hence $f \in K_{2}$.

Now suppose $\operatorname{orb}(x) \cap \operatorname{orb}(y)$ is nonempty, where $x \neq y$ are in $T(f)$. Again $f$ is conjugate to some $f^{\prime} \in K_{0}$. But the conjugacy must send points in $\operatorname{orb}(x)$ norb $(y)$ to points in the orbit of two turning points. This contradicts $f^{\prime} \in K_{0}$. Hence $f \in K_{3}$.

Thus $f \in K_{1} \cap K_{2} \cap K_{3}=K_{0}$. Q.E.D.

\section{REFERENCES}

1. R. Abraham, J. Robbin and A. Kelley, Transversal mappings and flows, Benjamin, New York, 1967. MR 39 \#2181.

2. Z. Nitecki, Nonsingular endomorphisms of the circle, Proc. Sympos. Pure Math., vol. 14, Amer. Math. Soc. Providence, R.I., 1970, pp. 203-220. MR 42 \#5275.

3. - Partitions for circle endomorphisms, C.U.N.Y. (preprint).

4. M. Shub, Endomorphisms of compact differentiable manifolds, Amer. J. Math. 91 (1969), 175-199.

5. S. Smale, Differentiable dynamical systems, Bull. Amer. Math. Soc. 73 (1967), 747-817. MR 37 \#3598.

6. M. V. Jakobson, On smooth mappings of the circle into itself, Mat. Sb. 85 (127) (1971), 163-188=Math. USSR Sb. 14 (1971), 161-185.

Department of Mathematics, Northwestern University, Evanston, Illinois 60201

Current address (Block and Franke): Institut des Hautes Études Scientifiques, 91Bures-sur-Yvette, France 